\title{
Axisymmetric Electromagnetic Wave Propagation Computation Using the Constrained Interpolation Profile Scheme with Large Time Steps
}

\author{
Jean Porto, Paul-Quentin Elias
}

\begin{abstract}
Conventional explicit time-domain methods used to solve Maxwell's equations are reliable and robust but are conditionally stable and often require the fulfilment of the condition CFL $\leq 1$. While this is acceptable for many applications, in some instances where the Maxwell's equations are solved alongside systems with slower propagation velocities, explicit methods prove costly. This is the case for non-relativistic electromagnetic Particle-In-Cell methods which are required to study plasma thrusters. Several algorithms have been proposed to retain a nearly explicit formulation using large time steps to achieve higher CFL values. Among these is the semi-Lagrangian Constrained Interpolation Profile method. While the ability of this method to handle CFL $>1$ has been demonstrated for planar 2D-3D cases, this has not been done for 2D cases with cylindrical symmetry. In this paper, a procedure is presented to compute the electromagnetic wave propagation in $2 \mathrm{D}$ domains with cylindrical symmetry using the Constrained Interpolation Profile (CIP) method. The CIP scheme is extended for CFL $\geq 1$ cases, and a ghost node method is proposed to deal with the axis singularity and with the wall boundary condition. The results are compared to the fields of a Hertzian dipole and with a coaxial cable, and they show a good agreement.
\end{abstract}

Index Terms-Constrained Interpolation Profile (CIP) method, electromagnetic wave propagation, 2D cylindrical coordinate system, Courant-Friedrichs-Lewy condition (CFL) condition.

\section{INTRODUCTION}

Finite-difference (FD) methods are one of the most extended and successful techniques for computational electromagnetic problems [1], [2]. One of the advanced techniques among the finite-difference methods is a stable third-order accurate and non-dissipative scheme initially developed in the field of Computational Fluid Dynamics [3], known as the Constrained Interpolation Profile (CIP) method. It is a semi-Lagrangian scheme which circumvent the Courant-Friedrichs-Lewy (CFL) stability condition [4], [5]. This feature allows computations with CFL values $\geq 1.0$, as can be seen in [6] and [7] where the authors performed simulations using a CFL value of 2.6 in a Cartesian coordinate system. It considers not only the electromagnetic fields, but also their spatial derivatives, therefore suppressing instabilities and providing lower numerical dispersion even when using coarse grids and large time steps [8]. It has been shown that it provides higher accuracy than the conventional finite-difference time-domain (FDTD) method

Jean Porto is with Sorbonne Université, Observatoire de Paris, PSL Research University, LERMA, CNRS UMR 8112, F-75005, Paris, France.

Jean Porto and Paul-Quentin Elias are with DPHY, ONERA, Université Paris Saclay, F-91123 Palaiseau - France (e-mail: jean_carlos.porto_hernandez@onera.fr ; paul-quentin.elias@onera.fr).
[9] under the condition of identical cell size [10]. A proof of the weak stability of the scheme is presented in [11].

The CIP scheme was combined with the Method of Characteristic (MoC) to get an accurate simulation of Maxwell's equations by Ogata et al. in 2006 [12]. Since then, several research developments have been published around the use of the CIP method for electromagnetics [13], [14]. Indeed, the CIP method has several interesting features for the computation of electromagnetic fields in time domain, among them its higher order means lower dispersion and lesser points required per wavelength. It can be used with variable cell sizes or subgridding techniques [15]. Finally, being a semiLagrangian technique, it can be used with larger CFL numbers than classical explicit FD techniques.

For the particular case of cylindrical systems, Tanaka et al. [13] proposed a method to deal with the radial terms with the CIP method. By considering that the computational domain can be seen as a medium with radially varying impedance, the characteristic variables can propagate radially with the semi-Lagrangian method taking into account the transmission and reflection of waves due to the impedance variation. We have tested this procedure and it has proved to be accurate for the treatment of electromagnetic problems in a cylindrical configuration. However, the procedure described by Tanaka et al. [13] is only limited to CFL $\leq 1.0$.

The CIP scheme features, coupled to the capacity to get accurate results in cylindrical coordinate systems, could be an interesting tool for the simulation of plasma propulsion devices as they can often be represented by an axisymmetric configuration [16]-[18]. An electromagnetic solver for cylindrical simulations based on the CIP method would have the advantages of updating the fields at the same grid location, which simplifies its integration to the Particle-In-Cell codes used to model these low pressure plasma sources [19], [20]. In addition, the characteristic form used in the CIP method for electromagnetic computation offers an easy handling of different types of boundary conditions such as a Perfectly Matched Layer (PML) and conducting or dielectric surfaces. Nevertheless, with the methodology described in [13], it will still be limited by the CFL $\leq 1.0$ condition.

In this paper we extend the results of Tanaka. et al. [13] to get a version of the 2D cylindrical CIP scheme that could be used with a CFL condition greater than one, thus providing a mean to considerably speed-up the calculations. In addition, we define a systematic strategy for the treatment of boundary conditions with this extended approach, using ghost nodes 
outside the computational domain to take into account the singularity at the symmetry axis in a cylindrical coordinate system and conducting surfaces.

To investigate the accuracy of the proposed scheme, we compared its results with those obtained using analytical expressions [21] to describe a Hertzian dipole. Another comparison seeking to test the stability of our approach was performed with the steady-state propagation of electromagnetic waves inside an open-ended coaxial wave guide. In that case the time-domain simulation with the CIP method is compared with the result using the COMSOL Multiphysics TM [22] software based on a frequency-domain finite-element analysis.

The paper is organized as follows. The sections II-A and II-B recall the main features of the CIP method adapted for 2D cylindrical systems. Then, section II-C extends the method proposed in [13] for cases where CFL $\geq 1$. Section II-D provides a simple method to account of the axis singularity and perfectly conducting boundaries in the frame of the CIP method with CFL $\geq 1$. Finally, section III considers two tests cases for the validation of the proposed method.

\section{THE CIP METHOD IN A TWO-DIMENSIONAL CYLINDRICAL COORDINATE SYSTEM}

\section{A. Fundamentals of the CIP Scheme}

Just as the finite-difference time-domain (FDTD) method, the CIP considers the electromagnetic fields on each grid point. However, it also propagates the spatial derivatives values for each field through an additional advection equation. This feature produces less numerical dispersion or instabilities [8] because for a given propagating wave, its values between the grid points are interpolated using not only the wave information at the grid but also its spatial derivatives. It allows to get a better approximation of the wave all over the computational domain and to maintain its original shape through the whole simulation. Consider the following advection equation for a one-dimensional problem :

$$
\frac{\partial f}{\partial t}+c \frac{\partial f}{\partial z}=0
$$

It represents the propagation of the a wavefield $f$ at a constant speed $c$. The CIP method solves not only eq. 1 , but also another differential equation for its spatial derivative along its propagation direction, obtained by differentiating eq. 1 :

$$
\frac{\partial g}{\partial t}+c \frac{\partial g}{\partial z}=0 \quad ; \quad g=\frac{\partial f}{\partial z}
$$

The advection phase may be better understood thanks to Fig. 1. It represents the field update process on the CIP method where at each time step, the value at any given grid point is updated using the value the field had before traveling up to that point the distance given by the time step and its propagating speed $(c \Delta t)$. It can be summarized as follows: consider a wave propagating along the positive direction of the $z$-axis $(+z)$ : the function $f$ and its spatial derivative $g$ at each time step $(\Delta t)$ and at each grid point $\left(z_{i}\right)$ are obtained by shifting its values from the position $z_{i}-c \Delta t$, where $c$ is the wave velocity. If the required values to be shifted are not on the grid points, they

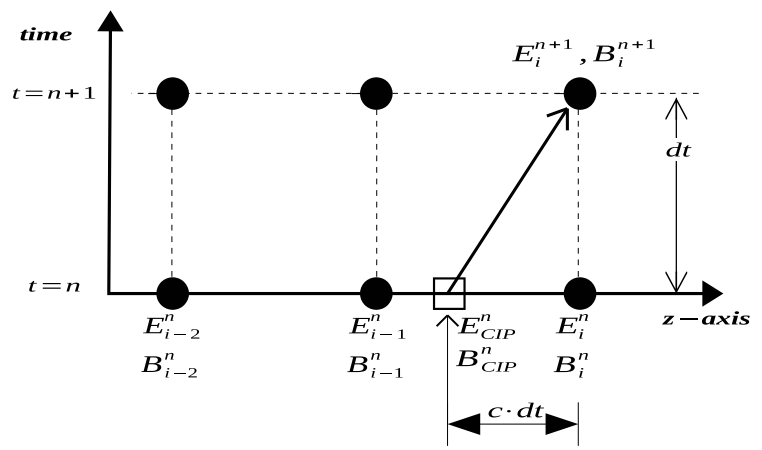

a)

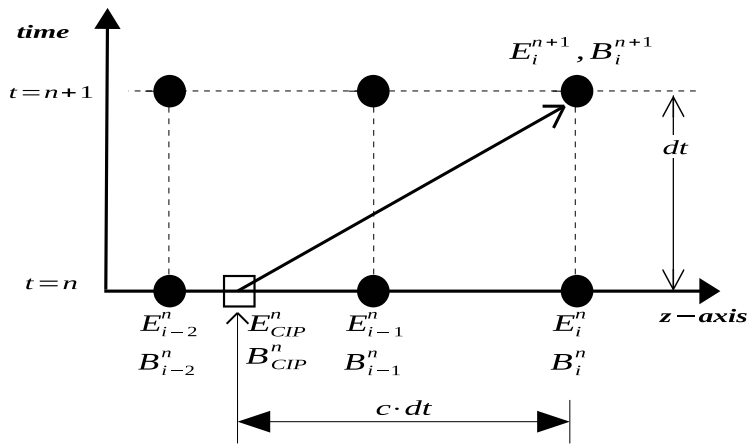

b)

Fig. 1. Fields update on the CIP method for the propagation of an electromagnetic wave where $E_{C I P}$ and $B_{C I P}$ are the interpolated fields between the grid cells: a) $\mathrm{CFL} \leq 1.0$, b) $1.0<\mathrm{CFL} \leq 2.0$.

are interpolated using a cubic polynomial function from the values at the endpoints of the cell, generating what we will call $F_{C I P}$ and $G_{C I P}$ located at the square marker as seen on Fig. 1. If we consider the right-propagating wave $(+z) f$ and $g$ at grid points $z_{i}$ and $z_{i-1}$, the interpolated values between those grid points are given by:

$$
\begin{aligned}
& F_{C I P}(z)=a_{i}\left(z-z_{i}\right)^{3}+b_{i}\left(z-z_{i}\right)^{2}+g_{i}\left(z-z_{i}\right)+f_{i} \\
& G_{C I P}(z)=\frac{\partial F(z)}{\partial z}=3 a_{i}\left(z-z_{i}\right)^{2}+2 b_{i}\left(z-z_{i}\right)+g_{i}
\end{aligned}
$$

Where $a_{i}$ and $b_{i}$ are coefficients calculated based on the functions $f$ and $g$ values at the grid points as follows:

$$
\begin{aligned}
a_{i} & =\frac{g_{i}+g_{i-1}}{(-\Delta z)^{2}}+\frac{2\left(f_{i}-f_{i-1}\right)}{(-\Delta z)^{3}} \\
b_{i} & =\frac{3\left(f_{i-1}-f_{i}\right)}{(-\Delta z)^{2}}-\frac{2 g_{i}+g_{i-1}}{(-\Delta z)}
\end{aligned}
$$

B. Electromagnetic Fields Numerical Analysis in the TwoDimensional Cylindrical Coordinate System

The Maxwell-Faraday and Maxwell-Ampère equations in a 2D axisymmetric cylindrical coordinate system can be written as follows if the medium is nonconducting:

$$
\begin{gathered}
\frac{\partial E_{r}}{\partial t}+c^{2} \frac{\partial B_{\varphi}}{\partial z}=-\frac{1}{\epsilon} J_{r} \\
\frac{\partial E_{z}}{\partial t}-c^{2} \frac{\partial B_{\varphi}}{\partial r}=\frac{c^{2}}{r} B_{\varphi}-\frac{1}{\epsilon} J_{z}
\end{gathered}
$$




$$
\frac{\partial E_{r}}{\partial z}-\frac{\partial E_{z}}{\partial r}+\frac{\partial B_{\varphi}}{\partial t}=0
$$

Where $\epsilon$ is the permittivity of the vacuum, $\mu$ is its permeability, the electric field has a radial and a longitudinal component, $E_{r}$ and $E_{z}$ respectively, and the magnetic field is oriented in the azimuthal direction $B_{\varphi}$. This set of equation can be expressed in matrix form as:

$$
\begin{gathered}
\frac{\partial \mathbf{W}}{\partial t}+\boldsymbol{\Lambda} \frac{\partial \mathbf{W}}{\partial r}+\boldsymbol{\Gamma} \frac{\partial \mathbf{W}}{\partial z}=\mathbf{S} \\
\mathbf{W}=\left[\begin{array}{c}
B_{\varphi} \\
E_{r} \\
E_{z}
\end{array}\right], \boldsymbol{\Lambda}=\left[\begin{array}{ccc}
0 & 0 & -1 \\
0 & 0 & 0 \\
-c^{2} & 0 & 0
\end{array}\right], \boldsymbol{\Gamma}=\left[\begin{array}{ccc}
0 & 1 & 0 \\
c^{2} & 0 & 0 \\
0 & 0 & 0
\end{array}\right] \\
\mathbf{S}=\left[\begin{array}{ll}
0, & -\frac{1}{\epsilon} J_{r}, \frac{c^{2}}{r} B_{\varphi}-\frac{1}{\epsilon} J_{z}
\end{array}\right]^{T}
\end{gathered}
$$

We now applied the change of variable proposed by Tanaka et al. [13] to get rid of the azimuthal magnetic field term in the right hand side of eq. 8: $I_{\varphi}=\frac{r}{\mu} B_{\varphi}$. The key idea here is to consider that the domain impedance is radially varying. Around a radial grid point, Tanaka et al. assume that the impedance is constant by part, thus removing the $1 / r$ dependence and transforming the medium as a graded-index medium. The final form of our system can be written as:

$$
\begin{gathered}
\mathbf{W}=\left[\begin{array}{c}
\frac{Z}{r_{j}} I_{\varphi} \\
E_{r} \\
E_{z}
\end{array}\right], \boldsymbol{\Lambda}=\left[\begin{array}{ccc}
0 & 0 & -c \\
0 & 0 & 0 \\
-c & 0 & 0
\end{array}\right], \boldsymbol{\Gamma}=\left[\begin{array}{ccc}
0 & c & 0 \\
c & 0 & 0 \\
0 & 0 & 0
\end{array}\right] \\
\mathbf{S}=\left[0,-\frac{1}{\epsilon} J_{r},-\frac{1}{\epsilon} J_{z}\right]^{T}
\end{gathered}
$$

Where $Z=\sqrt{\frac{\mu}{\epsilon}}$ and $r_{j}$ is the radial distance from the axis of symmetry to the grid point. We will treat the source term $\mathbf{S}$ as a non-advection term that will only be taken into account once the solution of the equation 8 is found under the hypothesis of $\mathbf{S}=0$. To do so, thanks to the change of variable previously introduced, we can now use the dimensional-splitting method [23]:

$$
\begin{array}{ll}
\frac{\partial \mathbf{W}}{\partial t}+\boldsymbol{\Lambda} \frac{\partial \mathbf{W}}{\partial r}=0 & \left(\mathbf{W}^{n} \rightarrow \mathbf{W}^{*}\right) \\
\frac{\partial \mathbf{W}}{\partial t}+\boldsymbol{\Gamma} \frac{\partial \mathbf{W}}{\partial z}=0 & \left(\mathbf{W}^{*} \rightarrow \mathbf{W}^{* *}\right)
\end{array}
$$

Where $\mathbf{W}^{n}$ is the field at time step $n, \mathbf{W}^{*}$ represents the field after propagation along the radial direction, and $\mathbf{W}^{* *}$ is the field after the propagation along the longitudinal direction. We will therefore express our system as the following set of propagating characteristics with its spatial derivatives along the propagation axis:

- Along the longitudinal direction (z-axis): $L_{p}$ and $L_{m}$

$$
\begin{gathered}
L_{p}=E_{r}+\frac{Z}{r_{j}} I_{\varphi} ; \quad L_{m}=E_{r}-\frac{Z}{r_{j}} I_{\varphi} \\
\frac{\partial L_{p}}{\partial z}=\frac{\partial E_{r}}{\partial z}+\frac{Z}{r_{j}} \frac{\partial I_{\varphi}}{\partial z} ; \quad \frac{\partial L_{m}}{\partial z}=\frac{\partial E_{r}}{\partial z}-\frac{Z}{r_{j}} \frac{\partial I_{\varphi}}{\partial z}
\end{gathered}
$$

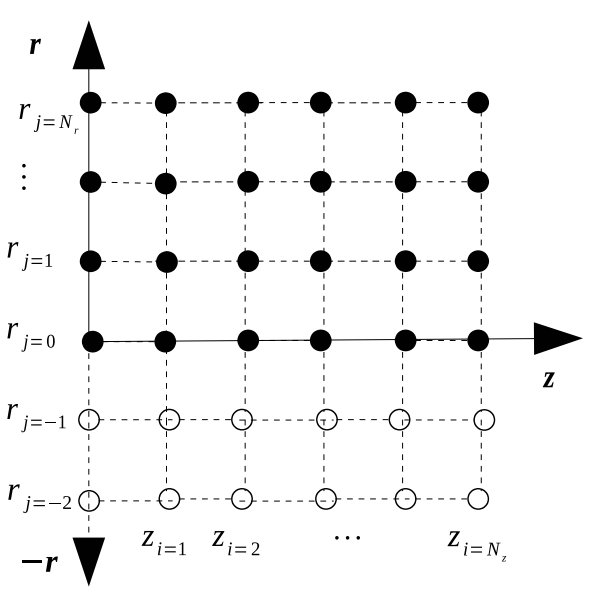

Fig. 2. Computational domain for the simulations in cylindrical coordinate systems. The colourless nodes represent ghost nodes outside the domain used for the boundary condition at the symmetry axis $Z$.

- Along the radial direction (r-axis): $R_{p}$ and $R_{m}$

$$
\begin{gathered}
R_{p}=E_{z}-\frac{Z}{r_{j}} I_{\varphi} ; \quad R_{m}=E_{z}+\frac{Z}{r_{j}} I_{\varphi} \\
\frac{\partial R_{p}}{\partial r}=\frac{\partial E_{z}}{\partial r}-\frac{Z}{r_{j}} \frac{\partial I_{\varphi}}{\partial r} ; \quad \frac{\partial R_{m}}{\partial r}=\frac{\partial E_{z}}{\partial r}+\frac{Z}{r_{j}} \frac{\partial I_{\varphi}}{\partial r}
\end{gathered}
$$

The subscript $p$ (plus) stands for a wave propagating in the positive direction of its axis (right-going wave), and $m$ (minus) in the negative direction (left-going wave).

Once equation 10 is solved, we can treat the non-advection term and obtain the field at the time step $n+l$ by adding the current source $\mathbf{J}$ as follows:

$$
\mathbf{E}^{n+1}=\mathbf{E}^{* *}-\frac{\Delta t}{\epsilon} \mathbf{J} \quad\left(\mathbf{W}^{* *} \rightarrow \mathbf{W}^{n+1}\right)
$$

\section{Wave Propagation with Multi-Layer Radial Scattering}

The computational domain used for our calculations in a 2D cylindrical coordinate configuration is presented in Fig. 2. The longitudinal axis goes from $z_{i=0}$ to $z_{i=N z}$, and the radial axis from $r_{j=0}$ to $r_{j=N r}$. The empty circles are ghost nodes helpful for the boundary conditions definition.

Thanks to the dimensional-splitting method presented in equations 9 and 10, the problem can be treated along each propagating axis. Regarding the longitudinal axis, the characteristic and its derivatives described in equations 11 and 12 are advected along the positive and negative direction of the $z$-axis using the interpolation polynomial described in section II-A and as seen in the schematic example of Fig. 1.

However, special treatment is required for the radial propagation because of the dependence of the $Z / r_{j}$ coefficient to the radial distance from the longitudinal axis. Tanaka et al. [13] has demonstrated that a radial scattering technique for the radial propagation produces accurate results. In order to extend this procedure for cases with $\mathrm{CFL} \geq 1.0$, let us begin by considering a right-going wave $R p$ propagating along the radial direction and a CFL $\leq 1.0$. For a given node position 


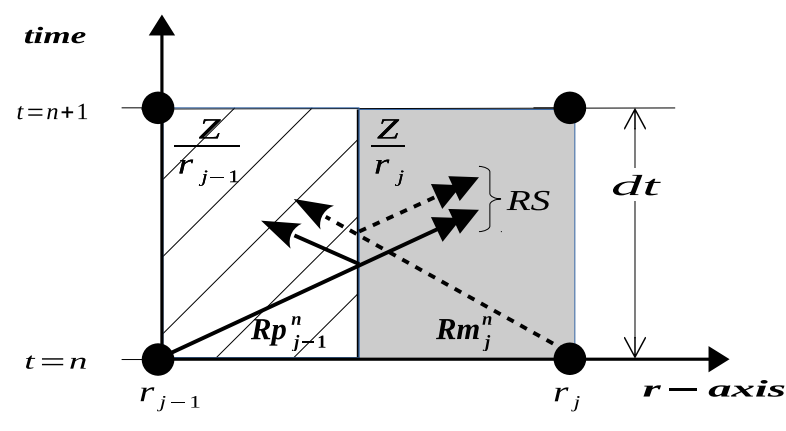

Fig. 3. Radial scattering procedure with $\mathrm{CFL} \leq 1.0$.

$\left(z_{i}, r_{j}\right)$ in the mesh, we can update its value at time step $n+1$ $\left(R p_{j}^{n+1}\right)$ based on $R p_{j-1}^{n}$ and $R p_{j}^{n}$. The space between two grid points (with radial distances to the symmetry axis $r_{j}$ and $r_{j-1}$ ) is considered as containing the interface between two different medias (located at $\Delta r / 2$ ) each one described by a fictitious impedance $\left(Z / r_{j}\right.$ and $\left.Z / r_{j-1}\right)$.

In Fig. 3, each medium is represented either as a grey zone or as a hatched one. At the interface between different media, as proposed by Okubo et al. in [24], the wave propagation across this interface can be treated using the standard electromagnetic interface conditions between two dielectrics. Thus, each incoming wave will be divided into a ' $R$ '-wave reflected back into the original media and a transmitted ' $\mathrm{T}$ '-wave that propagates to the second medium. We will therefore call radial scattering the procedure used to obtain the wave $R S$ which is the sum of a ' $\mathrm{T}$ ' wave coming from $r_{j-1}$ and an ' $\mathrm{R}$ '-wave coming from $r_{j}$. The resulting wave $R S$ can be thought of as an equivalent wave coming from the left in the same medium. This $R S$ wave is used to perform the CIP propagation. This variable $R S$ is shown in Fig. 3, and it is represented as the sum of the two double-arrow lines in the grey zone. It can be obtained as follows for a right-going radial propagating wave:

$$
\begin{aligned}
R S & =T_{+} R p_{j-1}^{n}+R_{-} R m_{j}^{n} \\
\frac{\partial(R S)}{\partial r} & =T_{+} \frac{\partial R p_{j-1}^{n}}{\partial r}+R_{-} \frac{\partial R m_{j}^{n}}{\partial r}
\end{aligned}
$$

With a transmission coefficient $T_{+}=\frac{2 r_{j-1}}{r_{j}+r_{j-1}}$ and a reflection coefficient $R_{-}=\frac{r_{j}-r_{j-1}}{r_{j}+r_{j-1}}$. We check that $T_{+}+R_{-}=1$. Similarly, for a left-going radial propagating wave:

$$
\begin{aligned}
R S & =T_{-} R m_{j+1}^{n}-R_{+} R p_{j}^{n} \\
\frac{\partial(R S)}{\partial r} & =T_{-} \frac{\partial R m_{j+1}^{n}}{\partial r}+R_{+} \frac{\partial R p_{j}^{n}}{\partial r}
\end{aligned}
$$

Where $T_{-}=\frac{2 r_{j+1}}{r_{j+1}+r_{j}}$ and $R_{+}=\frac{r_{j+1}-r_{j}}{r_{j+1}+r_{j}}$. As $r_{j} \rightarrow \infty$, $T \rightarrow 1$ and $R \rightarrow 0$, meaning for example that for a right going wave $R S \rightarrow R p_{j-1}$, which is equivalent to the classical CIP method in a planar Cartesian coordinate system.

Under the condition of CFL $>1.0$, a multi-layer radial scattering procedure is required in order to update the rightgoing characteristic $R p_{j}^{n+1}$. For this discussion we will restrict ourselves to the case CFL $\leq 2$, but the method described below

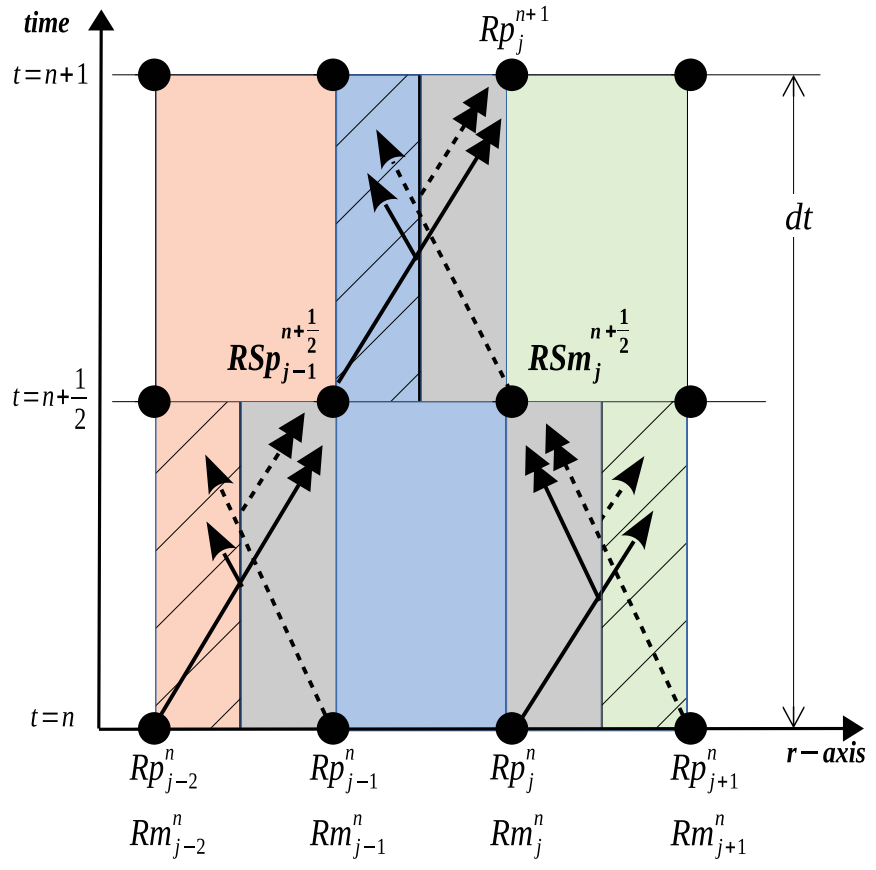

Fig. 4. Multi-layer scattering of the advected characteristics in the radial direction. Field update for a right-going wave $R p$ using $1.0<\mathrm{CFL} \leq 2.0$.

can be generalized to greater CFL numbers. We will use an intermediate stage described as $n+\frac{1}{2}$, as seen in Fig. 4, where the required propagating characteristics will be calculated with the following steps:

1) It starts with a radial scattering of the wave $R p_{j-2}^{n}$ through the medias with impedance $Z / r_{j-2}$ (hatched zone) and $Z / r_{j-1}$ (grey zone) using equations 16 . The sum of the transmitted part of $R p_{j-2}^{n}$ from the striped zone to the grey one (solid line with double-arrow), and the reflected part of $R m_{j-1}^{n}$ back into the grey zone (dashed line with double-arrow) is called $R S p_{j-2}$.

2) This right-going characteristic $R S p_{j-2}$ and the rightgoing wave $R p_{j-1}^{n}$ are both used to interpolate the characteristic value between $r_{j-2}$ and $r_{j-1}$ using eq. 3. The result of this CIP interpolation is shifted to the grid position $r_{j-1}$ and it is an intermediate-stage characteristic called $R S p_{j-1}^{n+\frac{1}{2}}$.

3) A radial scattering for the left-going wave $R m_{j+1}^{n}$ using equations 17 produces $R S m_{j+1}$. A CIP interpolation between $R S m_{j+1}$ and $R m_{j}^{n}$, and a shift of the result of this interpolation to the position $r_{j}$ allows us to get the intermediate-stage left-going wave $R S m_{j}^{n+\frac{1}{2}}$.

4) Finally, once the two intermediate characteristics are obtained ( $R S p_{j-1}^{n+\frac{1}{2}}$ and $R S m_{j}^{n+\frac{1}{2}}$ ), the radial scattering procedure is repeated with the right-going $\left(R S p_{j-1}^{n+\frac{1}{2}}\right)$ and the left-going $\left(R S m_{j}^{n+\frac{1}{2}}\right)$ characteristics between $t=n+\frac{1}{2}$ and $t=n+1$. The result of this scattering is shifted to $r_{j}$ and it gives us the update for $R p_{j}^{n+1}$.

Note that two interpolations are performed in this procedure, one of them is using $R S p_{j-2}$ and $R p_{j-1}^{n}$, and the other $R S m_{j+1}$ and $R m_{j}^{n}$. For a left-going wave, the entire pro- 
cedure is symmetrical, starting from the rightmost point $r_{j+2}$ and moving leftward to $r_{j}$ with the same multi-layer scattering.

This procedure can be extended to higher CFL numbers. First of all, the stencil changes as a function of the CFL number. The higher the CFL, the greater the stencil extent spreading over as many grid cells as the rounded upper value of the CFL number. Having defined the stencil scope, the steps 1 and 2 are performed for the leftmost grid nodes, then the radial scattering procedure is repeated for $R S p$ and $R S m$ over the remaining grid cells until the wave quantities reach $r_{j}$. For example, for a $\mathrm{CFL}=2.5$, the radial scattering is performed using a stencil scope going from $r_{j-3}$ to $r_{j+2}$.

\section{Boundary Conditions}

Different types of boundary conditions can be easily imposed with the use of ghost nodes as seen in Fig. 2. The main idea is to update the values of the ghost nodes based on the waves reaching the boundary, and then proceed to a propagation back into the computational domain. Thus, no special stencil is required for the boundary nodes which uses the same stencil as nodes in the domain. The rules for the ghost nodes update are the following:

\section{- Symmetry axis}

Based on [25], the ghost nodes of the symmetry axis (smaller than $r_{j=0}$ ) can be obtained by applying a simple transformation rule to the incoming wave: we multiply by -1.0 any radial derivative, and also any radial or azimuthal component of a vector quantity (letting the axial component unchanged). Under this rule, for any given real number $k$, we obtain:

$$
\begin{aligned}
E_{z}\left(z_{i}, r_{-k}\right) & =E_{z}\left(z_{i}, r_{k}\right) \\
\frac{\partial E_{z}\left(z_{i}, r_{-k}\right)}{\partial r} & =-\frac{\partial E_{z}\left(z_{i}, r_{k}\right)}{\partial r} \\
B_{\varphi}\left(z_{i}, r_{-k}\right) & =-B_{\varphi}\left(z_{i}, r_{k}\right) \\
\frac{\partial B_{\varphi}\left(z_{i}, r_{-k}\right)}{\partial r} & =\frac{\partial B_{\varphi}\left(z_{i}, r_{k}\right)}{\partial r}
\end{aligned}
$$

- Perfect Conductor (PEC) wall parallel to the z-axis Under the constraint of keeping a zero tangential electric field and normal magnetic field on a conducting wall located at $\left(z_{i}, r_{j}\right)$, we can impose the following conditions:

$$
\begin{aligned}
R_{p}\left(z_{i}, r_{j-k}\right) & =\alpha_{+} R_{m}\left(z_{i}, r_{j+k}\right)+\beta_{+} R_{m}\left(z_{i}, r_{j}\right) \\
R_{m}\left(z_{i}, r_{j+k}\right) & =\alpha_{-} R_{p}\left(z_{i}, r_{j-k}\right)+\beta_{-} R_{p}\left(z_{i}, r_{j}\right) \\
\alpha_{+} & =-\frac{\left(r_{j}+\Delta r\right)\left(2 r_{j}-\Delta r\right)}{\left(r_{j}-\Delta r\right)\left(2 r_{j}+\Delta r\right)} \\
\beta_{+} & =-\frac{2 r_{j} \Delta r}{\left(r_{j}-\Delta r\right)\left(2 r_{j}+\Delta r\right)} \\
\alpha_{-} & =-\frac{\left(r_{j}-\Delta r\right)\left(2 r_{j}+\Delta r\right)}{\left(r_{j}+\Delta r\right)\left(2 r_{j}-\Delta r\right)} \\
\beta_{-} & =+\frac{2 r_{j} \Delta r}{\left(r_{j}+\Delta r\right)\left(2 r_{j}-\Delta r\right)}
\end{aligned}
$$

Where the coefficients are obtained in order to ensure the $E_{z}=0$ condition over the wall with the interpolated fields: $\alpha_{+}$and $\beta_{+}$for a conducting wall at $r \leq r_{j}$ (eq. 19) receiving left-going waves $R_{m}$, and $\alpha_{-}$and $\beta_{-}$for a conducting wall at $r \geq r_{j}$ (eq. 20) receiving right-going waves $R_{p}$.

- Perfect Conductor (PEC) wall parallel to the r-axis

To keep a zero tangential electric field on the conducting wall, therefore ensuring $E_{r}=0$, we can impose the following conditions for any given real number $k$ to produce a mirrored wavefield with respect to the conducting wall:

$$
\begin{aligned}
E_{r}\left(z_{-k}, r_{j}\right) & =-E_{r}\left(z_{k}, r_{j}\right) \\
\frac{\partial E_{r}\left(z_{-k}, r_{j}\right)}{\partial z} & =\frac{\partial E_{r}\left(z_{k}, r_{j}\right)}{\partial z} \\
B_{\varphi}\left(z_{-k}, r_{j}\right) & =B_{\varphi}\left(z_{k}, r_{j}\right) \\
\frac{\partial B_{\varphi}\left(z_{-k}, r_{j}\right)}{\partial z} & =-\frac{\partial B_{\varphi}\left(z_{k}, r_{j}\right)}{\partial z}
\end{aligned}
$$

Once the ghost nodes have been updated, the corresponding characteristics can be computed on those locations, and its values can be propagated up to the inner nodes of the computational domain applying the same multi-layer radial scattering procedure previously described.

\section{NUMERICAL RESULTS}

\section{A. Hertzian Dipole}

We simulated the electromagnetic radiation of a Hertzian dipole with a 2D cylindrical coordinate system as seen in Fig. 2 , by imposing a Gaussian function for the source term $J_{z}$ along the symmetry axis with a width of $\sigma_{z}=2 \Delta z$ and $\sigma_{r}=$ $4 \Delta r$ where the mesh spacing $\Delta r=\Delta z$ is fixed to $40 \mu \mathrm{m}$. The dipole is located along the symmetry axis at $r=0$ and $z=z_{d}=0.5 * N_{z}$, as can be seen in Fig. 5, and it oscillates at a frequency of $300 \mathrm{GHz}$. The expression for $J_{z}$ is as follows (with $J_{0}=2487.75 \mathrm{~A} \cdot \mathrm{m}^{-2}$ ):

$$
J_{z}(r, z, t)=J_{0} \sin (\omega t) \exp \left(-\frac{r^{2}}{\sigma_{r}^{2}}-\frac{\left(z-z_{d}\right)^{2}}{\sigma_{z}^{2}}\right)
$$

Three different values of CFL were tested: $\mathrm{CFL}=1.0$, $\mathrm{CFL}=1.5$ and $\mathrm{CFL}=2.0$. The number of points per wavelength was 25 . The simulations results are compared with the analytical values calculated using the equations presented in Appendix A based on [21]. Figures 6 and 7 show a good agreement among the three cases with a longitudinal view along the line $\left(z=0 \rightarrow N_{z}, r=0.5 * N_{r}\right)$, and a radial view along $\left(z=0.25 * N_{z}, r=0 \rightarrow N_{r}\right)$. This position for the radial view was chosen over the alternative located at the middle point of the computational domain because the fields reach extremely high values in the zone close to the dipole. A radial view along the middle point of the computational domain would not be detailed enough to appreciate at the same time the two scales of the solution, close to the dipole and faraway from it.

In Fig. 5, we can also notice the presence of an absorbing boundary condition imposed in all the walls of the computational domain (except for the symmetry axis). Those absorbing layers can be seen in Fig. 6 and 7 as a yellow zone. The incoming waves into the Perfectly Matched Layer (PML) 
TABLE I

RELATIVE ERRORS FOR THE HERTZIAN DIPOLE SIMULATION.

\begin{tabular}{|l||c|c|c|}
\hline & $\mathrm{E}_{z}$ & $\mathrm{E}_{r}$ & $\mathrm{~B}_{\varphi}$ \\
\hline \hline Radial direction & $3.9 \%$ & $12.7 \%$ & $8.3 \%$ \\
\hline Longitudinal direction & $4.5 \%$ & $12.8 \%$ & $5.7 \%$ \\
\hline
\end{tabular}

show an attenuation until the fields completely drop to zero as desired. The implementation of this boundary condition was done based on [26] with a linearly increasing attenuation parameter $k_{f}$ that depends on the distance from the PML starting point, and it is zero outside that layer. This attenuation parameter is treated as a non-advection term for equation 8 , and it is taken into account in the same way as the source term $\mathbf{S}$ in eq. 15. For example, after the advection phase, the following equation is applied to the right-propagating $L_{p}$ characteristic:

$$
L_{p(i, j)}^{n+1}=L_{p(i, j)}^{n+1}\left(1.0-\Delta t k_{f}\right)
$$

Where $k_{f}=k_{f_{\max }}\left(\frac{z_{i}-z_{o}}{z_{p m l}}\right) . k_{f_{\max }}$ is the maximum value of the attenuation parameter, $z_{o}$ is the starting point of the PML, and $z_{p m l}$ is the total length of the layer. The same procedure is applied to the other outbound characteristics, adapting the $k_{f}$ coefficient for each direction. One should also underline that the equations being in characteristic form, the implementation of the PML is straightforward and does not require a modified stencil.

A constant offset between the analytical results and the plots from the CIP simulations can be seen in Fig. 6 at $z=0$. An explanation to this offset comes from the fact that the analytical expressions used for this comparison describe the radiation from the oscillation of two point charges in free space separated by an infinitesimally small distance. The numerical representation of this infinitesimal nature of the Hertzian dipole is limited by the size of the mesh spacing. Therefore, since a better match between the simulation and the theoretical results can be achieved with either a smaller mesh spacing or a greater number of points per wavelength, the accuracy obtained with the previously described simulation parameters is deemed sufficient for a proper comparison point. The relative error along a given section compared to the analytical result is given by:

$$
\eta(F)=\frac{\sum_{i}\left(F_{i}^{A}-F_{i}^{C I P}\right)^{2}}{\sum_{i}\left(F_{i}^{A}\right)^{2}}
$$

Where $F=E_{r}, E_{z}, B_{\varphi}, F^{A}$ and $F^{C I P}$ are the analytical and the simulation results, respectively. The results can be seen in Table I. The overlapping of all the plots obtained with the simulations using three different CFL values is a sign of the robustness and precision of the multi-layer scattering procedure even for larger CFL.

\section{B. Steady-State Open-Ended Coaxial Cable}

Another comparison was performed using the simulation of an open-ended coaxial wave guide in vacuum, as shown in Fig. 5. This test case has been chosen because it is

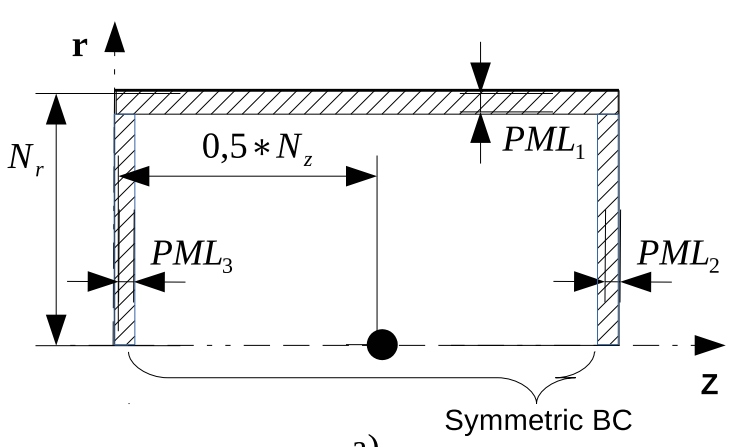

a)

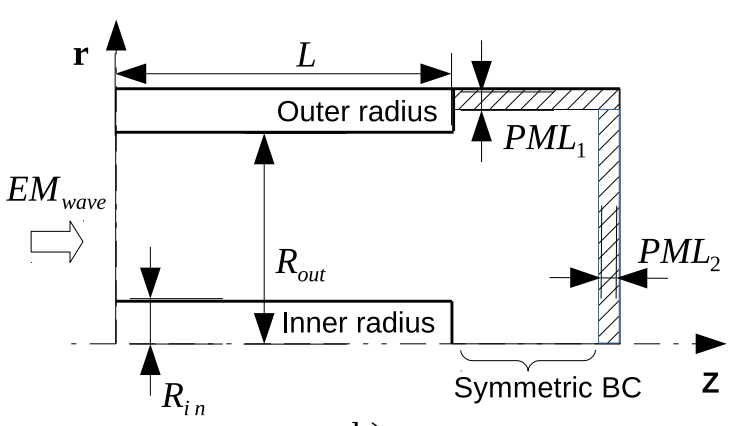

b)

Fig. 5. Computational domain for a) the Hertzian dipole, b) the coaxial cable.
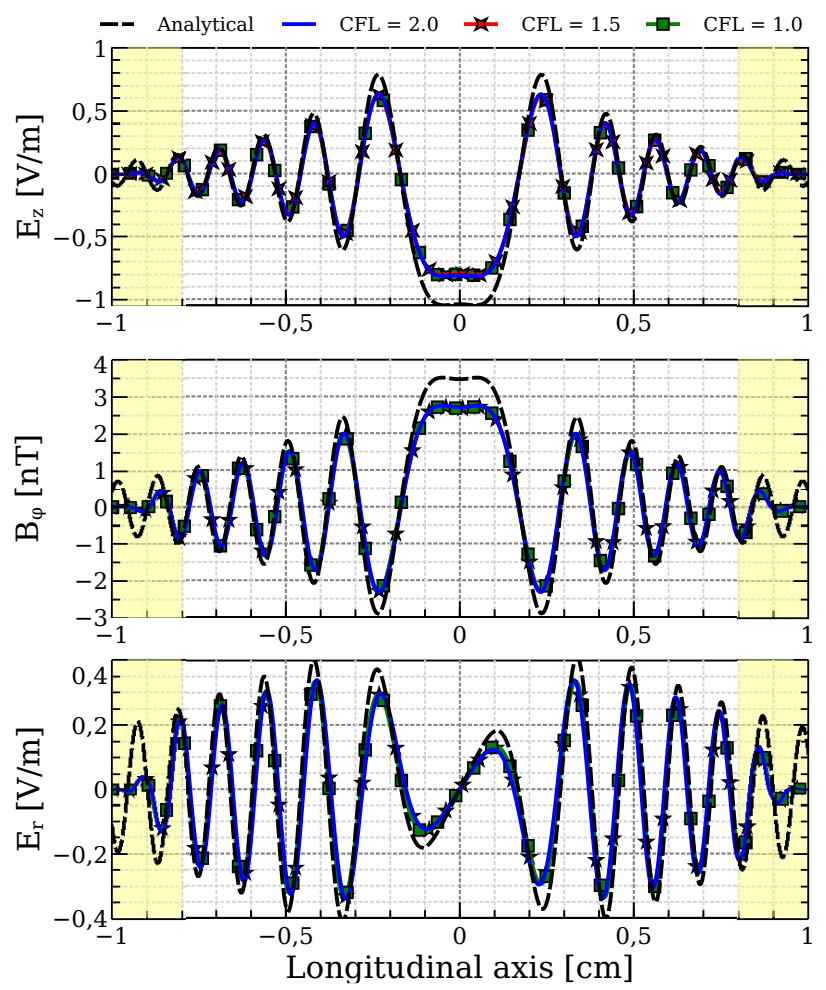

Fig. 6. Electromagnetic radiation of a Hertzian dipole in the longitudinal direction. Comparison among the exact results from an analytical model and different values of CFL (1.0, 1.5, and 2.0). Longitudinal electric field (top), azimuthal magnetic field (middle), and radial electric field (bottom). 

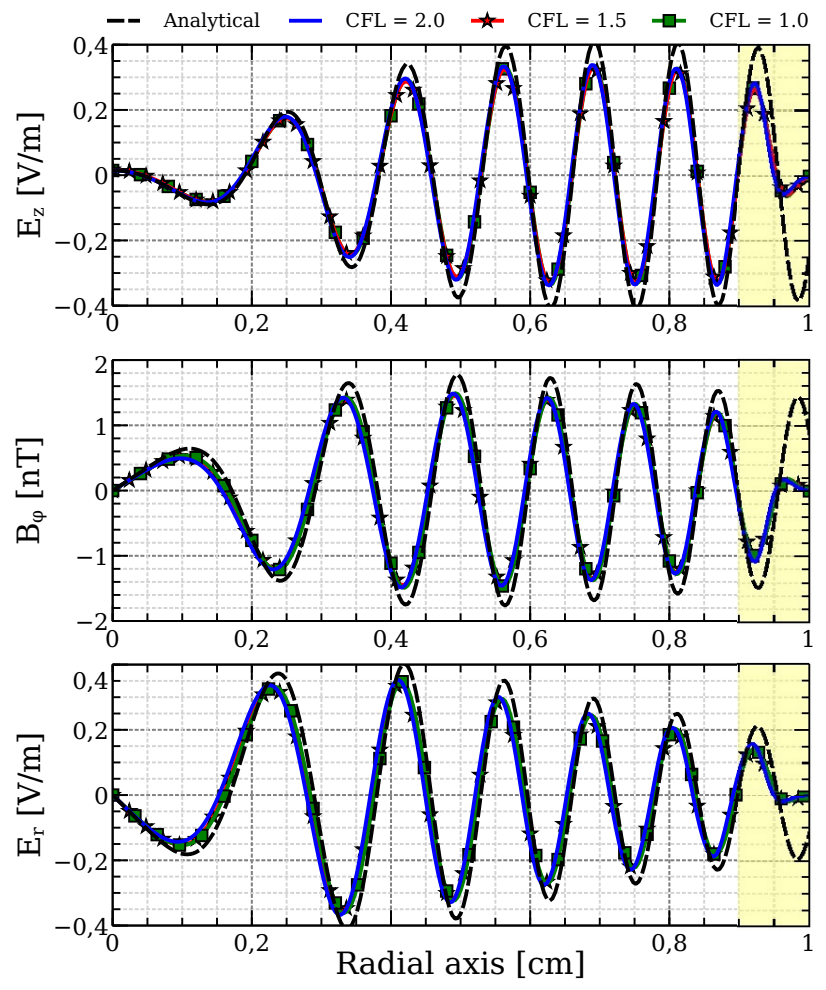

Fig. 7. Electromagnetic radiation of a Hertzian dipole in the radial direction. Comparison among the exact results from an analytical model and different values of CFL (1.0, 1.5, and 2.0). Longitudinal electric field (top), azimuthal magnetic field (middle), and radial electric field (bottom).

representative of the coaxial configuration used in an ECR plasma thruster for which this model is developed [18], [20]. The dimensions of the coaxial wave guide are: an inner radius $R_{\text {in }}=1.15 \mathrm{~mm}$, an outer radius of $R_{\text {out }}=13.75 \mathrm{~mm}$, a length $L=200 \mathrm{~mm}$, and a rectangular computational domain of $z=400 \mathrm{~mm}$ and $r=100 \mathrm{~mm}$. A $2.45 \mathrm{GHz}$ Transverse Electro-Magnetic (TEM) input wave is imposed at the lefthand wall. More precisely, the analytic solution for a right propagating coaxial TEM mode was used to set the rightpropagating characteristics $L_{p}$ and $\partial_{z} L_{p}$ in eq. 12 at the left of the simulation domain boundary. The exiting left-going characteristic $L_{m}$ was left free. For the boundary conditions we used a PML at the right-hand side and at the top right corner, and a perfectly conducting surface boundary condition at the inner and outer radius. A constant mesh spacing of 0.23 $\mathrm{mm}$ was used. The simulation is run for 3800 iterations, which corresponds to more than two round trips of the incident wave, to establish a standing wave in the waveguide.

The standing wave pattern is retrieved for the CIP simulation by taking the results once an amplitude peak in reached for the time-varying stationary wave. As a comparison, the electric field obtained using a Frequency Domain electromagnetic solver from a commercial software is compared to the wave pattern obtained with the CIP method. The same computational domain is used, using a triangulation of characteristic length of $0.2 \mathrm{~mm}$. The electromagnetic fields amplitudes are compared to the standing wave envelope obtained from the CIP method, as shown in Fig. 8. In addition,
TABLE II

POWER BUDGET FOR THE OPEN-ENDED COAXIAL CABLE.

\begin{tabular}{|l||c|c|c|}
\hline & CIP & $\begin{array}{c}\text { Frequency } \\
\text { Domain }\end{array}$ & $\begin{array}{c}\text { Analytic } \\
{[27]}\end{array}$ \\
\hline \hline Input power [W] & 200.0 & 200.0 & 200.0 \\
\hline Reflected power [W] & 198.5 & 198.5 & 198.4 \\
\hline Radiated power [W] & 1.5 & 1.5 & 1.6 \\
\hline
\end{tabular}
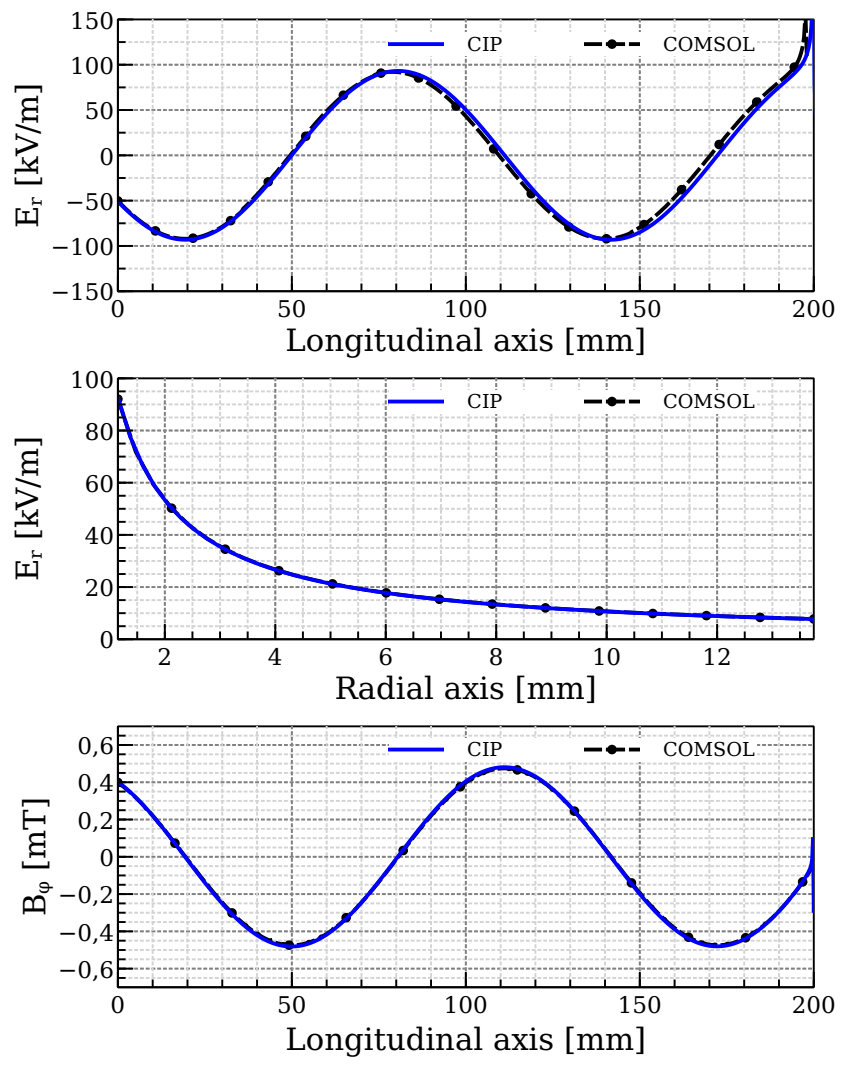

Fig. 8. Electromagnetic fields in a coaxial cable simulated using the CIP method and the COMSOL Multiphysics ${ }^{\mathrm{TM}}$ [22] software based on the finiteelement method with a CFL $=1.0$. The radial cross section is taken at $z=80 \mathrm{~mm}$, and the longitudinal cross section at the outer surface of the inner conductor.

the reflected and radiated power are compared as well, as shown in Table II. In the limit of large wavelength compared to the coaxial diameter $\lambda \gg R_{\text {out }}$, an analytic formula for the radiated power exists as presented in Appendix B, and it is included for comparison [27].

A good agreement is found between the two cases. The relative error between the CIP radial electric field and the frequency domain result does not exceed $0.1 \%$. The radiated power obtained with the CIP method also matches the predicted value from the frequency-domain solver and the analytic formulation. This fact give us confidence about the stable nature of the solution obtained by our procedure. Our time-domain solution successfully reproduced the steady state solution obtained with a frequency-domain finite-element method. 


\section{CONCLUSION}

We have proposed a procedure for computing the propagation of electromagnetic waves in axisymmetric $2 \mathrm{D}$ domains using the Constrained Interpolation Profile scheme. The main contribution of this development is the extension of the radialscattering algorithm for CFL conditions greater than 1.0 in cylindrical configurations, as well as a simple treatment of boundary conditions for the symmetry axis and conducting walls. For this work, we did not exceed a CFL condition of 2.0 , but in principle higher values may be achieved. In the future, our goal is to explore the CFL limit until which the procedure can be used based on a Von Neumann stability analysis. In addition, the CIP scheme described above will be applied to 2D cylindrical simulations of an ECR plasma source [20] where the source terms (charge density and plasma current) will be obtained using a Particle-In-cell code. In this case where a strong coupling between the plasma and the electromagnetic fields exists, the divergence conservation properties of the scheme are important and will be assessed. In particular, the need for a divergence cleaning method will be tested. If such were the case, one should note that the Method of Characteristic used in this approach enable a straightforward extension to hyperbolic divergence cleaning methods [29].

\section{APPENDIX A}

\section{EXPLICIT EXPRESSIONS FOR THE ELECTROMAGNETIC FIEldS OF A HERTZIAN Dipole}

Explicit expressions for the electromagnetic fields of an oscillating z-directed dipole $\mathbf{p}(t)=p \hat{\mathbf{z}} \cos (\omega t)$ were derived and presented in [21] in spherical coordinates. Here $\mathbf{r}$ is the vector pointing to the observation point from the dipole and $\theta=\angle(\mathbf{r}, \mathbf{z})$ is the angle with the dipole axis.

$$
\begin{aligned}
H_{\phi}(\mathbf{r})= & {\left[-k \cos (k r-\omega t)+\frac{\sin (k r-\omega t)}{r}\right]\left[\frac{\sin \theta}{4 \pi r}\right] p \omega } \\
E_{r}(\mathbf{r})= & {\left[k \sin (k r-\omega t)+\frac{\cos (k r-\omega t)}{r}\right]\left[\frac{2 \cos \theta}{4 \pi \epsilon_{0} r^{2}}\right] p } \\
E_{\theta}(\mathbf{r})= & {\left[k \sin (k r-\omega t)+\frac{\cos (k r-\omega t)}{r}\right]\left[\frac{\sin \theta}{4 \pi \epsilon_{0} r^{2}}\right] p } \\
& -\frac{p k^{2} \sin \theta}{4 \pi \epsilon_{0} r} \cos (k r-\omega t)
\end{aligned}
$$

These expressions were transformed to a cylindrical coordinate system in order to perform the comparison with the simulations results obtained using the CIP method. The next formulas are used for the radial and longitudinal electric field:

$$
\begin{aligned}
& E_{r}(r, z)=E_{r}(\mathbf{r}) \sin \theta+E_{\theta}(\mathbf{r}) \cos \theta \\
& E_{z}(r, z)=E_{r}(\mathbf{r}) \cos \theta-E_{\theta}(\mathbf{r}) \sin \theta
\end{aligned}
$$

\section{APPENDIX B}

\section{ANALYTICAL FORMULA FOR THE RADIATED POWER FROM AN OPEN-ENDED COAXIAl CABle}

In [27], we can find the following analytical expression for the radiated power $P_{\text {out }}$ from an open-ended coaxial cable:

$$
P_{\text {out }}=P_{\text {in }} \frac{k^{4}\left(R_{\text {out }}^{2}-R_{\text {in }}^{2}\right)^{2}}{12 \sqrt{\epsilon} \ln \left(\frac{R_{\text {out }}}{R_{\text {in }}}\right)}
$$

The formula is a function of the input power $P_{i n}$, the inner $R_{\text {in }}$ and outer $R_{\text {out }}$ radius, and the wave number $k$.

\section{ACKNOWLEDGMENT}

This work was made in the framework of the project MINOTOR that has received funding from the the European Union's Horizon 2020 research and innovation program under grant agreement No 730028.

\section{REFERENCES}

[1] Shiraishi, K., and Matsuoka, T. "Wave Propagation Simulation Using the CIP Method of Characteristic Equations." Communications in Computational Physics, Vol. 3, No. 1, 2008, pp. 121-135.

[2] Merino, M., Sanchez-Villar, A., Ahedo, E., Bonoli, P., Lee, J., Ram, A., and Wright, J. "Wave Propagation and Absorption in ECR Plasma Thrusters." 35th International Electric Propulsion Conference, Atlanta, USA, 2017.

[3] T. Yabe and T. Aoki, A universal solver for hyperbolic equation by cubicpolynomial interpolation, I. One-dimensional solver, Comput. Phys. Commun., 66 (1991), 219-232.

[4] T. Yabe, F. Xiao and T. Utsumi, The constrained interpolation profile method for multiphase analysis, J. Comput. Phys., 169 (2001), 556-593.

[5] P.K.Smolarkiewicz and J.A.Pudykiewicz,'A Class of Semi- Lagrangian Approximations for Fluids,'J. Atmospheric Sciences, Vol.49, pp.20282096, 1992.

[6] Nie, Y., Fu, K., and Lv, X. "CIP Method of Characteristics for the Solution of Tide Wave Equations." Advances in Mathematical Physics, Vol. 2018, 2018. doi:10.1155/2018/3469534.

[7] Tachioka, Y., Yasuda, Y., and Sakuma, T. "Application of the Constrained Interpolation Profile Method to Room Acoustic Problems: Examination of Boundary Modeling and Spatial/Time Discretization." Acoustical Science and Technology, Vol. 33, No. 1, 2012, pp. 21-32. doi:10.1250/ast.33.21.

[8] Kajita, K., Baba, Y., Nagaoka, N., and Ametani, A. "Computation of Lightning Electromagnetic Pulses Using the Constrained Interpolation Profile Method." Electric Power Systems Research, Vol. 115, 2014, pp. 94-101. doi:10.1016/j.epsr.2014.02.017.

[9] K. S. Yee, "Numerical solution of initial boundary value problems involving Maxwell's equations in isotropic media," IEEE Trans. Antennas Propag., vol. AP-14, no. 4, pp. 302-307, May 1966.

[10] K. Okubo and N. Takeuchi, "Analysis of an electromagnetic field created by line current using constrained interpolation profile method," IEEE Trans. Antennas Propag., vol.55, no.1, pp.111-119, Jan. 2007.

[11] Tanaka Daiki. Stability Analysis of the CIP Scheme and its Applications in Fundamental Study of the Diffused Optical Tomography. PhD thesis, Kyoto University Graduate School of Informatics, 2014.

[12] Ogata, Y., Yabe, T., and Odagaki, K. "An Accurate Numerical Scheme for Maxwell Equations with CIP-Method of Characteristics". Communications in Computational Physics. Vol 1. No. 2, 2006, pp. 311-335.

[13] Tanaka, Y., Baba, Y., Nagaoka, N., and Ametani, A. "Computation of Lightning Electromagnetic Pulses with the Constrained Interpolation Profile Method in the 2-D Cylindrical Coordinate System.” IEEE Transactions on Electromagnetic Compatibility, Vol. 56, No. 6, 2014, pp. $1497-1505$.

[14] Chakarothai, J., Chen, Q., and Sawaya, K. "Three-Dimensional Electromagnetic Scattering Analysis Using Constrained Interpolation Profile Method." IEICE Transactions on Communications, Vol. E93-B, No. 10, 2010, pp. 2619-2628.

[15] Kobayashi, S., Suzuki, Y., and Baba, Y. "Lightning Electromagnetic Field Calculation Using the Constrained Interpolation Profile Method with a Subgridding Technique." IEEE Transactions on Electromagnetic Compatibility, Vol. 58, No. 5, 2016, pp. 1682-1685. doi:10.1109/TEMC.2016.2575079.

[16] Domínguez, Adrián \& Cichocki, Filippo \& Merino, Mario \& Fajardo, Pablo \& Ahedo, Eduardo. (2018). Axisymmetric plasma plume characterization with 2D and 3D particle codes. Plasma Sources Science and Technology. 27. 10.1088/1361-6595/aae702. 
[17] Szabo, James \& Warner, Noah \& Martinez-Sanchez, Manuel \& Batishchev, Oleg. (2014). Full Particle-In-Cell Simulation Methodology for Axisymmetric Hall Effect Thrusters. Journal of Propulsion and Power. 30. 197-208. 10.2514/1.B34774.

[18] F. Cannat, T. Lafleur, J. Jarrige, P. Chabert, P.-Q. Elias, and D. Packan, "Optimization of a coaxial electron cyclotron resonance plasma thruster with an analytical model," Phys. Plasmas, vol. 22, no. 5, p. 053503 , 2015.

[19] C. K. Birdsall and A. B. Langdon, Plasma Physics via Computer Simulation. Philadelphia, IOP Publishing, 1991.

[20] Jean C. Porto, Paul-Quentin Elias. "Full-PIC Simulation of an ECR Plasma Thruster with Magnetic Nozzle". International Electric Propulsion Conference 2019, Sep. 2019, Vienne, Austria.

[21] Orfanidis, S. J. Chapter 15: "Radiation Fields", in "Electromagnetic Waves and Antennas.", Vol. 2, No. Rutgers University, 2004, pp. 313-321. doi:10.1016/B978-075064947-6/50011-3.

[22] COMSOL Multiphysics® v. 5.4. www.comsol.com. COMSOL AB, Stockholm, Sweden.

[23] Macnamara, S., and Strang, G. "Splitting Methods in Communication, Imaging, Science, and Engineering." 2016, pp. 1-21. doi:10.1007/9783-319-41589-5.

[24] Okubo, K., Yoshida, Y., and Takeuchi, N. "Consideration of Treatment of the Boundary between Different Media in Electromagnetic Field Analysis Using the Constrained Interpolation Profile Method." IEEE Transactions on Antennas and Propagation, Vol. 55, No. 2, 2007, pp. 485-489. doi:10.1109/TAP.2006.889984.

[25] Mohseni, K., and Colonius, T. "Numerical Treatment of Polar Coordinate Singularities." Journal of Computational Physics, Vol. 157, No. 2, 2000, pp. 787-795. doi:10.1006/jcph.1999.6382.

[26] Ishizuka, T., and Okubo, K. "Formulation and Examination of the Perfectly Matched Layer for Sound Field Analyses Using the Constrained Interpolation Profile Method." Acoustical Science and Technology, Vol 34, No. 5, 2013, pp. 378-381. doi:10.1250/ast.34.378.

[27] Schelkunoff, S. A "On Diffraction and Radiation of Electromagnetic Waves" Physical Review, Vol. 56, pp. 308-316,1939.

[28] Ogata, Y., Yabe, T., Takizawa, K., and Ohkubo, T. "The Analysis of Electromagnetic Waves Using CIP Scheme with Soroban Grid." Computational Fluid Dynamics 2004, No. 3, 2006, pp. 141-146. doi:10.1007/3540-31801-1-16.

[29] C.-D. Munz, P. Ommes, R. Schneider, "A three-dimensional finitevolume solver for the Maxwell equations with divergence cleaning on unstructured meshes", Computer Physics Communications, Volume 130, Issues 1-2,2000, pp. 83-117, doi:10.1016/S0010-4655(00)00045-X.

[30] Huang, W., Sloan, D. M. (1993). Pole condition for singular problems: the pseudospectral approximation. Journal of Computational Physics, 107(2), 254-261.

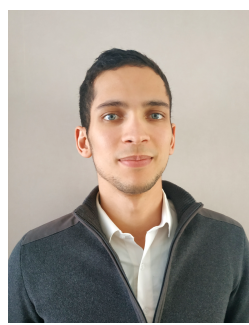

Jean Porto received the B.S. degree in mechanical engineering from the Universidad del Norte in Barranquilla, Colombia, and the M.S. degree specialized in aerospace engineering from the Arts et Métiers Engineering School in Paris, France. He used to work as a simulation engineer using finite element analysis for the automotive industry. He is currently pursuing a Ph.D. degree in physics at the French National Aerospace Research Centre (ONERA) and the Paris Observatory. His research interests include computational plasma physics for electric thrusters and numerical methods for partial differential equations.

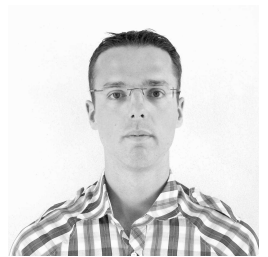

Paul-Quentin Elias holds a M.S degree in applied physics from Ecole Centrale Paris, France (2003). $\mathrm{He}$ earned a $\mathrm{PhD}$ in Energetics in 2007, from the same institution. He is a Senior Research Scientist at the French National Aerospace Research Center (ONERA). 